\title{
Modeling and Control of Robot Manipulators with the Constraints at the Moving Objects
}

\author{
Felix Kulakov*, Gennadiy V. Alferov ${ }^{\dagger}$, Polina Efimova ${ }^{\ddagger}$, Svetlana Chernakova ${ }^{\S}$, and Dmitry Shymanchuk ${ }^{\mathbb{\top}}$ \\ Saint-Petersburg State University, 7/9 Universitetskaya nab., St. Petersburg, 199034, Russia \\ Email: *kufelix@yandex.ru, ${ }^{\dagger}$ alferovgv@gmail.com, ${ }^{\ddagger}$ yefimovapa@gmail.com,

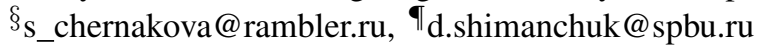

\begin{abstract}
In this paper a method of constructing a model of control over compliant motion of robot manipulators is considered. In this method the elastic deformations of flexible manipulators elements are used as feedback signals.
\end{abstract}

\section{INTRODUCTION}

At the moment there exist a lot of methods for constructing a system of force-torque control over compliant motion of robot manipulators. These methods are generally focused on solving the problems of using robots to automate the assembly and other operations which require interaction between robot and mechanically constrained objects [1]. Nevertheless, application of these methods in practice has not given the desired results. It is related to the fact that the applied approaches do not allow to construct the force-torque control system with the good enough dynamic properties. This negates the effectiveness of robots and economic justifiability of robot-based applications. In addition, the methods being in use differ in dependences of the dynamic properties on the parameters variations of control system and controlled object. It should be also noted that there is poor effectiveness in use of the pointed control laws in case of robot manipulators with flexible elements, in particular with flexible segments mostly used in space robotics [2]-[5]. The

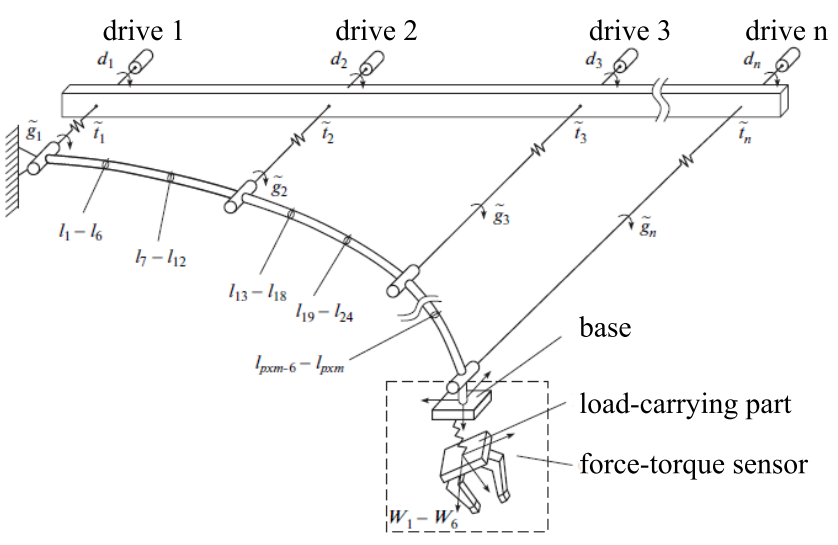

Fig. 1. Scheme of the manipulator with elastically deformable segments and transmission

proposed approach to construction of an active force-torque control system is based on the use of the elastic deformations of the flexible manipulator's elements as feedback signals, rather than the traditional forces and reaction moments that occur while contacting of gripper with being moved objects [1]. Control laws as the functions of the feedback data are proposed to be built so that they could generate potential and quasi-potential control forces for the joints of the robot manipulator. It is also important the minimum sum of original and artificial potential energies of the system "manipulator object" to be in the target point or on the program trajectory of the manipulator. The stability conditions of the controlled process imply that for any variation of the control law's parameters the forces would stay potential. In this case, the manipulator being applied only potential forces and dissipative forces of friction will have a stable balance in the target point or on the program trajectory [6]-[9]. The proposed method is applicable to any real robot manipulators with elastic compliance in the joints.

\section{DYNAMIC MODEL OF ROBOT MANIPULATOR}

The scheme of the manipulator equipped with direct current drives is shown in Fig. 1. The current position of the manipulator equipped with controlled drives is defined by the joint coordinates $\tilde{g}_{i}(i=1,2, \ldots, n)$. They are the angles of rotation or linear displacement of one $i$-segment relative to the $(i-1)$-th segment. These coordinates can be combined into an $n$-dimensional vector $\tilde{g}=\left(\tilde{g}_{1}, \tilde{g}_{2}, \ldots, \tilde{g}_{n}\right)^{T}$ of joint coordinates.

Joint coordinates change is performed with the use of drives with the current state determined by $n$-dimensional vector of drives coordinates $d=\left(d_{1}, d_{2}, \ldots, d_{n}\right)$. Its components are the rotation angles of the electric motors shafts. Communication between these vectors is represented by the relation:

$$
\tilde{g}=g+\tau, \quad g=P(d),
$$

where $g$ is $n$-dimensional vector of the joints rotation angles of the shaft motors drives, $\tau=\left(\tau_{1}, \tau_{2}, \ldots, \tau_{n}\right)$ is $n$-dimensional vector of the shaft gear's elastic deformations, $P(d)$ is $n$-dimensional vector-function of the reduction transformations.

Suppose that manipulator segments are elastically deformable, i.e. flexible. To take this into account in the models the finite element method is used. In other words, each of segments is approximated by the chain of $k$ solid bodies which are connected to each other by elastic elements of zero dimensions and zero masses. Moreover, the elastic deformation of each segment is defined by coordinates $l_{1}, l_{2}, \ldots, l_{6 \times k}$, 
which are the elastic deformations quantities of all $k$ elastic elements approximating the segment. Deformation of all $n$ parts is defined by the coordinates of all $(6 \times k \times n)$ elastic elements approximating these segments and integrated into vector $l=\left(l_{1}, l_{2}, \ldots, l_{6 \times k \times n}\right)$. The load-carrying structure of force-torque sensor gives elastic deformations presented by six wrist coordinates of vector $w=\left(w_{1}, w_{2}, w_{3}, w_{4}, w_{5}, w_{6}\right)$. Thus, the current state of the manipulator is determined by vector $\tilde{q}=(\tilde{g}, d, g, \tau, l, w)$. However, since $n$-dimensional vectors $\tilde{g}, g, \tau, d$ are related by equations (1), description of current state for the manipulator is sufficient to include two of these vectors, for example, $g$ and $\tau$. Then the vector of manipulator state is used $q=(g, \tau, l, w)=(g, e)$, where $e=(\tau, l, w)$ is elastic deformation vector of all the flexible manipulator elements. The dimension of the vector $q$ is equal to $n+m$, the dimension $e$ equals $m=n+6 \times k \times n+6$.

The constraints imposed on the movable objects which are rigidly grasped by manipulator gripper, can be described by $r$ algebraic equations $(r \leq 6)$ :

$$
M(X)=0 ; \quad X=\left(y_{1}, y_{2}, y_{3}, \theta_{1}, \theta_{2}, \theta_{3}\right)=L(g, e),
$$

where $M(X)$ is $r$-dimensional continuously differentiable vector function, $X$ is 6-dimensional vector of gripping position, which determines coordinates $y_{1}, y_{2}, y_{3}$ for position of its characteristic point in the fixed coordinate system and the Euler rotation angles $\theta_{1}, \theta_{2}, \theta_{3}$ for the coordinate system of gripping relative to the stationary coordinate system.

Equation (2) can be transformed into the following system:

$$
Q(g, e)=M[L(g, e)]=0 .
$$

This system relates coordinates of state vector $q=(g, e)$. System (3) is a system of $r$ equations for holonomic mechanism constraints. This system allows presenting $r$ vector components in an explicit form whereas the components are integrated into the vector $g^{r}$ in the function of all the remaining independent coordinates of vector $q=(g, e)$

$$
g^{r}=\Phi\left(g^{n-r}, e\right)=\Phi(\bar{q}),
$$

where $g^{n-r}$ is $(n-r)$-vector composed of $(n-r)$ component of vector $g$ that are not included in $g^{r}, \bar{q}=\left(g^{n-r}, e\right)$ is $(n-r+m)$-vector of independent generalized coordinates.

The state vector $q$ may be presented through $\bar{q}$ by using (4) as following:

$$
q=\left(g^{r}, g^{n-r}, e\right)=(\Phi(\bar{q}), \bar{q}) .
$$

The dynamic model is represented by two subsystems of equations. The first one describes the behavior of the mechanical manipulation part:

$$
E(q, \dot{q}, \ddot{q})=\frac{d}{d t}\left(\frac{\partial L}{\partial \dot{q}}\right)-\frac{\partial L}{\partial q}=H F_{a}+F_{d i s}+S^{T} \lambda .
$$

The second subsystem

$$
U=L_{a} \dot{I}_{a}+R_{a} I_{a}+k_{s} \dot{d}, \quad f_{a}=k_{n} I_{a},
$$

describes the processes in the electric circuits of direct current motors. In this model the following notation is used: $F_{a}$ is $n$-dimensional vector of generalized control forces assigned to the vector $g, F_{d i s}=K_{F} \dot{q}$ and $S^{T} \lambda$ are $(n+m)$-vectors of generalized dissipative forces and generalized reactions assigned to the vector $q, K_{F}$ is symmetric positively defined $(n+m) \times(n+m)$-matrix of friction coefficients, $E(q, \dot{q}, \ddot{q})$ is Euler operator from the Lagrange function $L=T-P, T$ and $P$ are kinematic and potential energies of the manipulator with

drives, $\lambda$ is $r$-dimensional Lagrange multiplier, $H^{T}=[E: 0]$ is block $n \times(n+m)$-matrix, $E$ is unity $n \times n$-block, $I_{a}$ is $n$-dimensional current vector for armatures of motor electric drives $R_{a}, L_{a}$ are diagonal $n \times n$-matrices of active resistance and armature windings inductance, $U$ is $n$-dimensional vector of voltages applied to the armature windings, which perform the control, $f_{a}$ is $n$-dimensional vector of generalized control forces assigned to the vector $d ; k_{s}$ and $k_{n}$ are diagonal $n \times n$ matrices of transformation of rotative speed vector for motors armatures into back electromotive force (EMF) and armatures current vector $I_{a}$ into the vector $f_{a}, Z=\partial P(d) / \partial d$ is $n \times n$ matrix of reduction.

Differential normalized form of constraint equations obtained by differentiating system (2), has the following form:

$$
\hat{n} \dot{X}=0 \text {, }
$$

where $\hat{n}=R^{-1}(\partial M / \partial X)$ is normalized $(r \times 6)$-matrix of constraints, $R=\left\{R_{1}, R_{2}, \ldots, R_{r}\right\}$ is diagonal $(r \times r)$-matrix; $R_{i}$ is Euclidean norm of $i$ th matrix row.

The following statements are true for $\dot{X}$ :

$$
\dot{X}=\dot{X}_{r}+\dot{X}_{e}=S \dot{\bar{q}}, \quad \dot{X}_{r}=J_{r} \dot{g} \quad \text { and } \quad \dot{X}_{e}=J_{e} \dot{e}
$$

where $\dot{X}_{r}$ and $\dot{X}_{e}$ are the velocity vectors of gripping displacement generated by vectors and $\dot{g}$ and $\dot{e}$ respectively, since $J_{r}, J_{e}$ and $S$ are $6 \times n, 6 \times m, 6 \times(n+m)$-Jacobians.

Then it follows from (8) that

$$
\hat{n} S \dot{q}=0, \quad \hat{n} J_{r} \dot{q}=-\hat{n} J_{e} \dot{e}, \quad \dot{q}^{r}=-S_{I}^{-1} S_{I I} \dot{\bar{q}}
$$

where: $S_{I}$ and $S_{I I}$ are $r \times r$ and $(n-r \times m)$-blocks of the matrix $S$ with the rank $r$. The velocity vector of manipulator state changing $\dot{q}=\left(\dot{q}^{r}, \dot{\bar{q}}\right)$ can be presented through the velocity vector $\bar{q}$ of independent generalized coordinates changing as following:

$$
\dot{q}=\left(\dot{q}^{r}, \dot{\bar{q}}\right)=W \dot{\bar{q}}, \quad W=\left[-\underline{S}_{I}^{-1} \underline{S}_{I I} \underline{I}-\right.
$$

where $W=(n+m) \times(n-r+m)$ is matrix of rank $(n-r+m)$, $I$ is a unity matrix.

Subsystem (1) with $(n+m)$ equations, subsystem (2), (3) with $2 n$ equations and subsystem (10) with $r$ constraint equations consist of $(n+m+2 n+r)$ variables integrated into vectors $\bar{q}, F_{a}, L_{a}, \lambda$.

These subsystems are transformed into the system of $(2 n+$ $m-r)$ differential equations with the following subsystems:

$$
\begin{aligned}
& \dot{\bar{q}}_{1}=-A^{-1}\left[\left(B+K+K^{*}\right) \dot{\bar{q}}_{1}+C \dot{\bar{q}}+D-W^{T} H F_{a}\right] \\
& \dot{\bar{q}}=\bar{q}_{1}, \quad \varepsilon \dot{F}_{a}=-T_{*} F_{a}-k_{t} U-k_{n} T_{*} R_{a}^{-1} k_{s} Z^{-1} H^{T} W \dot{\bar{q}}_{1} .
\end{aligned}
$$


In these subsystems the described further notation is used: $A=W^{T} a W$ positively defined symmetric $a-(n+m-$ $r) \times(n+m-r)$ - and $(n+m) \times(n+m)$-matrices of inertia for the manipulator with drives, assigned to the vectors $\bar{q}$ and $q$, respectively, $B=\partial A / \partial t-0,5 \partial\left(\dot{\bar{q}}^{T} A\right) / \partial \bar{q}$ and $K=\bar{W}^{T} K_{F} \bar{W}$ are $(n-r+m) \times(n-r+m)$-matrix for representing centrifugal and Coriolis forces manipulator elements and positively defined symmetric matrix of friction coefficients. $C=\left[\begin{array}{c:c}0 & 0 \\ \hdashline 0 & C_{e}\end{array}\right]$ and $C_{e}=\left[\begin{array}{c:c:c}C_{\tau} & 0 & 0 \\ \hdashline 0 & C_{e} & 0 \\ \hdashline 0 & 0 & e\end{array}\right]$ are positively semidefined and defined symmetric matrices of rigidity, $C_{\tau}, C_{l}, C_{w}$ are rigidity matrices of redactors, segments and wrist

$$
T_{0}=L_{a} R_{a}^{-1}, \quad T_{x}=\left\|T_{0}\right\| T_{0}^{-1}, \quad K_{t}=K_{I I} T_{0} R_{0}^{-1} .
$$

The obtained system of $2 n-r+m$ differential equations relative to the same amount of variables integrated into vectors $\bar{q}$ and $F_{a}$, appears as the dynamic model of the manipulator. Its peculiarity is that some of the equations from subsystem (12) included in this system has a very small value of the scalar parameter $\varepsilon$ at the derivative of the variable $F_{a}$, which is resulted from dynamic properties of the electrical manipulator drives.

\section{Method OF ACTIVE CONTROL}

The system (11), (12) satisfies Tikhonov's theorem [10]. In particular, its singularly perturbed subsystem (12) has an isolated root at $\varepsilon=0$ :

$$
F_{a}^{0}=T_{*}^{-1} k_{t} U-T_{*}^{-1} k_{t} k_{s} Z^{-1} H^{T} W \dot{\bar{q}}_{1} .
$$

And this root is a stable stationary point of adjoint subsystem obtained from (12) if the vector $\left(\bar{q}, \bar{q}_{1}\right)$ is considered to be a parameter such as zero.

Indeed, the adjoint subsystem presented in deviations has the following form: $\varepsilon \Delta F_{a}=-T_{x} F_{0}^{a}$, where $\Delta F_{a}=F_{a}-F_{a}^{0}$. This corresponds to system of $n$ isolated first order equations describing damped aperiodic, which means stable processes. According to Tikhonov's theorem, this allows using a generative system instead of initial system (11), (12) as a dynamic model of the manipulator. This is important for analysis of its dynamic properties, in particular the stability of the forcetorque control process. The system is formed by replacing vector $F_{a}$ with vector $F_{a}^{0}$ presented by expression (13) in initial system. This leads to the following simplified form of the dynamic model which can be presented as a system of $(n-r+m)$ second order equations

$$
E_{*}(\bar{q}, \dot{\bar{q}}, \ddot{\bar{q}})=A \ddot{\bar{q}}+\left(B+K+K^{*}\right) \dot{\bar{q}}+C \bar{q}+D=\bar{W}^{T} H k_{t} U,
$$

where $K^{*}=\bar{W}^{T} H k_{n} R_{a}^{-1} k_{s} Z^{-1} H \bar{W}$ is $(n-r+m)(n-$ $r+m)$-symmetric positively defined matrix of electromagnetic friction coefficients. The matrix is assigned with vector $\bar{q}$.

The main terms in the used control laws are given in the table below. In these laws $k_{p}$ and $k_{G}$ are $6 \times 6$-matrices of gripping position intensification and artificial rigidity, $x_{d r}$ and $x_{r}$ are vectors of desired and current positions of the manipulator operating tool, $G_{d}$ and $G=\left(J_{w}^{T}\right)^{-1}\left(C_{w} w+\partial \Pi_{w} / \partial w\right)$ are vectors of desired and current constraint reactions values

\begin{tabular}{|c|c|}
\hline Method & The main terms from control laws \\
\hline Rigidity control & $k_{t} U=J^{T} k_{p}\left(x_{d r}-x_{r}+k_{G} G\right)$ \\
\hline The explicit force control & $U=j^{T} k_{p}\left(G-G_{d}\right)$ \\
\hline $\begin{array}{c}\text { The hybrid position- } \\
\text {-force control }\end{array}$ & $\begin{array}{c}k_{t} U=J^{T}\left(I-\hat{n}^{T} \hat{n}\right) k_{p}\left(x_{d r}-x_{r}\right)+ \\
+J^{T} k_{G} \hat{n}^{T} \hat{n}\left(G-G_{d}\right)\end{array}$ \\
\hline Impedance control & $k_{t} U=J^{T} k_{p}\left(x_{d r}-x_{r}+k_{G} G\right)$ \\
\hline
\end{tabular}

presented in the system of gripping coordinates, $\partial \Pi_{w} / \partial w$ is the weight of the gripping.

The first term of force $G$ is defined by using a wrist forcetorque sensor from the measured values of elastic deformations $w$, the second one is calculated with the use of measured coordinates of vector $\bar{q}$, while the components of vector $l$ defining the elastic deformations are usually not taken into account because of their smallness.

The new approach proposed in the paper assumes replacing the vectors $G$ and $G_{d}$ from the table of the control laws by vectors $X_{e}$ and $X_{d e}$ of the current and desired gripping motions. Here the vectors $X_{e}$ and $X_{d e}$ are generated by the vectors of the current and desired elastic deformations of manipulator structure. The substitution is hold with taking into account that for small increments $\Delta e=\left(e_{d}-e\right)$ and $\Delta g=\left(g_{d}-g\right)$ in (9) and (10) the terms $\Delta g$ and $\Delta e$ may be used instead of $\dot{g}$ and $\dot{e}$. Then $J_{r} \Delta g=J_{z}\left(g_{d}-g\right)=x_{d_{r}}-x_{r}$, $J_{e} \Delta e=J_{e}\left(e_{d}-e\right)=x_{d_{e}}-x_{e}$ and $\hat{n} J_{r}\left(g_{d}-g\right)=$ $-\hat{n} J_{e}\left(e_{d}-e\right)$.

In this case the system (14) has the following form:

$$
E_{0}(\bar{g}, \dot{\bar{g}}, \ddot{\bar{g}})=R\left(\bar{q}_{d}-\bar{q}\right),
$$

For the law analogical to the hybrid, rigidity and explicit force control law:

$$
\begin{gathered}
R=W^{T} H J_{r}^{T}\left[k_{g} \hat{n}^{T} \hat{n}+k_{p}\left(E-\hat{n}^{T} \hat{n}\right) J_{e} H^{T} W\right] . \\
R=W^{T} H J_{r}^{T}\left[k_{g} \hat{n}^{T} \hat{n}+k_{p} J J_{r} H^{T} W\right] . \\
R=W^{T} H J_{r}^{T} k_{g} n^{T} n J_{r} H^{T} W .
\end{gathered}
$$

It is obvious that $R$ is a symmetric positively defined matrix, which follows from its structure. Therefore $R\left(\bar{q}_{d}-\bar{q}\right)$ is a potential force while determining $\bar{q}$ in the neighborhood close to the vector $\bar{q}_{0}$.

To obtain the conditions of stability for the compliant motion control system when using control laws derived above, variable $\bar{\Delta}=\bar{q}-\bar{q}_{0}(t)$ is introduced. This variable is a deviation of trajectory $\bar{q}(t)$ from trajectory $\bar{q}_{0}(t)$ of steady motion. Then assuming that the steady process is quasistationary, i.e. $\bar{q}_{0}(t) \approx$ const, $\dot{\bar{q}}_{0}(t) \approx 0$ gives the system in the linearized form:

$$
A_{0} \ddot{\bar{\Delta}}+\left(K_{0}+K_{0}^{*}\right) \dot{\bar{\Delta}}+\left(P_{0}+C_{0}+R_{0}\right) \bar{\Delta}=0 .
$$

Matrices $A_{0}, K_{0}, K_{0}^{*}, P_{0}=\partial D / \partial \bar{\Delta}$ are constant values of corresponding matrices $A, K, K^{*}, P, R$ at argument value $\bar{q}$ of their elements equaled to $\bar{q}_{0} \approx$ const. Matrix $b_{0}=(\partial A / \partial \bar{q}) \dot{\bar{q}}$ vanishes at $\dot{\bar{q}}_{0}(t)=0$.

It is important to note that $\dot{\bar{q}}_{0}(t)=0$ are symmetric positively defined matrices, as well as $(n+m-r) \times\left(n_{m}-r\right)$-matrix $\left(C_{0}+R_{0}\right)$, which follows from the structure $C_{0}$ presented in 
the notes to the equations (11) for $C_{0}$ and the structure $R_{0}$ presented in the expressions (16), (17), (18).

Here the possibility of achieving the stability "in the large" process $\bar{\Delta}(t)$ described by obtained system (19) is tested. For that purpose the following function is used as Lyapunov function:

$$
V=\frac{1}{2} \dot{\bar{\Delta}}^{T} A_{0} \dot{\bar{\Delta}}+\frac{1}{2} \bar{\Delta}^{T}\left(P_{0}+C_{0}+R_{0}\right) \bar{\Delta},
$$

which vanishes at the point of equilibrium, i.e. at $\bar{\Delta}=\dot{\bar{\Delta}}=0$ and shows positive definiteness. This follows from the positive definiteness of $A_{0}$, as well as $\left(P_{0}+C_{0}+R_{0}\right)$. The last statement is due to the positive definiteness of symmetric matrix $\left(R_{0}+C_{0}\right)$, which can be increased indefinitely by increasing coefficients $k_{g}$ and $k_{p}$ and by increasing the rigidity matrix of the manipulator construction $C_{e}$. For this reason it appears possible to suppress the effect of the symmetric, but not necessarily positively defined Hessian $\partial^{2} \Pi_{w} / \partial \Delta^{2}=P_{0}$, to make $\left(P_{0}+C_{0}+R_{0}\right)$ positively defined. The time derivative of $V$ due to the equation (19) has the form

$\dot{V}=\dot{\bar{\Delta}}^{T} A_{0} \ddot{\bar{\Delta}}+\dot{\bar{\Delta}}^{T}\left(P_{0}+C_{0}+R_{0}\right) \bar{\Delta}=-\dot{\bar{\Delta}}^{T}\left(K_{0}+K_{0}^{*}\right) \dot{\bar{\Delta}}$.

This function is a negative definite function of vector $\dot{\bar{\Delta}}$ and semidefinite function of vector $(\bar{\Delta}, \dot{\bar{\Delta}})$ vanishing at $\dot{\bar{\Delta}}=0$. It follows from the above that being analyzed system is stable, and that the point $\bar{\Delta}=0$ and $\dot{\bar{\Delta}}$ the unique point of stable equilibrium, since if $\bar{\Delta} \neq 0, \dot{\bar{\Delta}}=0$, then in accordance with (19) $A_{0} \ddot{\bar{\Delta}} \neq 0$, i.e. $\ddot{\bar{\Delta}} \neq 0$, and any point $\bar{\Delta} \neq 0, \dot{\bar{\Delta}} \neq 0$ can not be stable.

Therefore, not only is there a stable equilibrium at the point $\bar{\Delta}=0, \dot{\bar{\Delta}}=0$ that is actually asymptotic. This means that the real parts of all the characteristic equation roots in system (19) are negative. In other words there are neither roots with positive real part, nor and imaginary ones among all the roots.

It is also very important to note that the only condition for sustainability is a symmetry positive definiteness of matrix artificial rigidity matrix $k_{g}$ and intensification matrix $k_{p}$ in control laws, as well as permutability with the matrix $\hat{n}^{T}$, which makes $\bar{R}$ positive semi-definite. The magnitude of these matrices is not limited. If the term dependent from $\dot{\bar{q}}$ is used in the control law, then this term must included linearly as $K_{v} \dot{\bar{q}}$, where $K_{v}$ is a symmetric positive semidefinite matrix. There are no any limits for the parameters characterizing manipulator structure. Since symmetry and positive definiteness of matrix $R$ in equation (15) do not depend on the steady trajectory $q_{0}=q_{0}(t)$ or equilibrium point $q_{0}=$ const, the proof of asymptotic stability holds true for any point from position acceptable region of the manipulator.

It is useful to note that calculating the program value of vector $X_{d e}$ and measuring its current value $X_{e}=X_{r}+X_{l}+$
$X_{w}$ used in the obtained control laws instead of traditional programmed and current values $G_{d}$ and $G$ of reaction forces, is performed as follows: $X_{d}=C_{M}^{-1} G_{d}$, where $G_{d}$ and the rigidity of manipulator construction $C_{M}$ are known in advance. To obtain $X_{e}=X_{r}+X_{l}+X_{w}$, only values of $X_{r}$ and $X_{l}$ must be measured, as $X_{w}=0$ due to absence of wrist forcetorque sensor. The value of $X_{r}$ is generated by using strain gage sensors measuring the value of elastic torsion for output shafts of joint drives. It appears to be more complex to measure the value of bending for the segments of manipulator arms. For this purpose optical techniques may be used.

\section{CONCLUSION}

The paper presents a new approach to the force-torque control over robot manipulators in case of contact with stationary surfaces of the environment while performing service operations. This approach proposes using control laws of such a structure that provides the manipulator drives to transform the joint control forces to the form of artificial potential forces. In the paper the new way for transforming the structures of these control laws is presented.

\section{REFERENCES}

[1] F. M. Kulakov, "Active force-torque robot control without using wrist force-torque sensors," J. Comput. Syst. Sci. Int., vol. 51, no. 1, pp. 147$168,2012$.

[2] G. V. Alferov et al., "Game-theoretic model of inspection by anticorruption group," in Proceedings of the International Conference on Numerical Analysis and Applied Mathematics 2014, ICNAAM 2014, Rhodes, Greece, 2015, vol. 1648, art. no. 450009.

[3] O. Malafeyev et al., "Group strategy of robots in game-theoretic model of interception with incomplete information," in 2015 International Conference on Mechanics - Seventh Polyakhov's Reading, St. Petersburg, Russia, 2015, art. no. 7106751.

[4] G. V. Alferov et al. "Programming the robot in tasks of inspection and interception," in 2015 International Conference on Mechanics - Seventh Polyakhov's Reading, St. Petersburg, Russia, 2015, art. no. 7106713.

[5] F. Kulakov et al. "Methods of remote control over space robots," in 2015 International Conference on Mechanics - Seventh Polyakhov's Reading, St. Petersburg, Russia, 2015, art. no. 7106742.

[6] P. Efimova and D. Shymanchuk, "Dynamic model of space robot manipulator," Appl. Math. Sci., vol. 9, no. 93-96, pp. 4653-4659, 2015.

[7] A. Shmyrov and D. Shymanchuk, "Maneuvering in near-Earth space with the use of the collinear libration points," in 2015 International Conference on Mechanics - Seventh Polyakhov's Reading, St. Petersburg, Russia, 2015, art. no. 7106777.

[8] A. Shmyrov et al. "Prospects for the use of space robots in the neighbourhood of the libration points," Appl. Math. Sci., vol. 8, no. 49-52, pp. 2465-2471, 2014.

[9] F. Kulakov et al., "Supervisory remote control of space robot in an unstable libration point," in 2013 IEEE 7th International Conference on Intelligent Data Acquisition and Advanced Computing Systems, IDAACS 2013, Berlin, Germany, vol. 2, pp. 925-928, 2013, art. no. 6663062.

[10] A. N. Tihonov, "Differential equations systems containing small parameters in fromt of derivatives," (In Russian), Math. Sbornik, vol. 31(73), no. 3, pp. 575-586, 1952. 